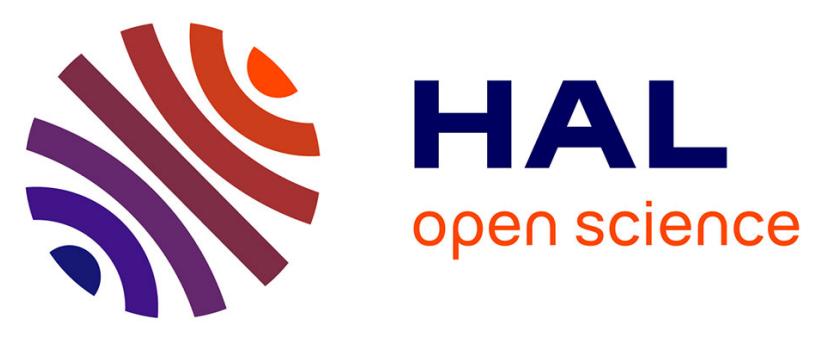

\title{
Moderate Certainty Evidence Suggests the Use of High-Flow Nasal Cannula Does Not Decrease Hypoxia When Compared With Conventional Oxygen Therapy in the Peri-Intubation Period
}

Dipayan Chaudhuri, David Granton, Dominic Xiang Wang, Sharon Einav, Yigal Helviz, Tommaso Mauri, Jean-Damien Ricard, Jordi Mancebo, Jean-Pierre Frat, Sameer Jog, et al.

\section{- To cite this version:}

Dipayan Chaudhuri, David Granton, Dominic Xiang Wang, Sharon Einav, Yigal Helviz, et al.. Moderate Certainty Evidence Suggests the Use of High-Flow Nasal Cannula Does Not Decrease Hypoxia When Compared With Conventional Oxygen Therapy in the Peri-Intubation Period. Critical Care Medicine, 2020, 48 (4), pp.571-578. 10.1097/CCM.0000000000004217 . hal-02436055

\author{
HAL Id: hal-02436055 \\ https://hal.science/hal-02436055
}

Submitted on 1 Jul 2020

HAL is a multi-disciplinary open access archive for the deposit and dissemination of scientific research documents, whether they are published or not. The documents may come from teaching and research institutions in France or abroad, or from public or private research centers.
L'archive ouverte pluridisciplinaire HAL, est destinée au dépôt et à la diffusion de documents scientifiques de niveau recherche, publiés ou non, émanant des établissements d'enseignement et de recherche français ou étrangers, des laboratoires publics ou privés. 


\title{
Moderate Certainty Evidence Suggests the Use of High-Flow Nasal Cannula Does Not Decrease Hypoxia When Compared With Conventional Oxygen Therapy in the Peri-Intubation Period: Results of a Systematic Review and Meta-Analysis
}

\author{
Dipayan Chaudhuri, $\mathrm{MD}^{1}$; David Granton, BSc ${ }^{1}$; Dominic Xiang Wang, $\mathrm{BSc}^{2}$; Sharon Einav, $\mathrm{MD}^{3,4}$; \\ Yigal Helviz, $\mathrm{MD}^{3}$; Tommaso Mauri, MD ${ }^{5,6}$; Jean-Damien Ricard, MD,8; Jordi Mancebo, MD; \\ Jean-Pierre Frat, $\mathrm{MD}^{10,11,12}$; Sameer Jog, $\mathrm{MD}^{13}$; Gonzalo Hernandez, MD ${ }^{14}$; Salvatore M. Maggiore, $\mathrm{MD}^{15}$; \\ Carol Hodgson, $\mathrm{PhD}^{16}$; Samir Jaber, $\mathrm{MD}^{17}$; Laurent Brochard, MD ${ }^{18}$; Karen E. A. Burns, MD ${ }^{18}$; \\ Bram Rochwerg, $\mathrm{MD}^{1,19}$
}

'Department of Medicine, McMaster University, Hamilton, ON, Canada. ${ }^{2}$ Schulich School of Medicine, Western University, London, ON, Canada. ${ }^{3}$ General Intensive Care Unit, Shaare Zedek Medical Center, Jerusalem, Israel.

${ }^{4}$ Faculty of Medicine, Hebrew University, Jerusalem, Israel.

${ }^{5}$ Dipartimento di fisopatologia medico-chirurgica e dei trapianti, Università degli Studi di Milano, Milan, Italy.

${ }^{6}$ Department of Anesthesia, Critical Care and Emergency, Fondazione IRCCS Ca' Granda Ospedale Maggiore Policlinico, University of Milan, Milan, Italy.

${ }^{7}$ Assistance Publique-Hôpital de Paris, Service de Réanimation Médicochirurgicale, Hôpital Louis Mourier, Colombes, France.

${ }^{8}$ Université Paris Diderot, IAME, UMR 1137, Sorbonne Paris Cité, Paris, France.

${ }^{9}$ Servei de Medicina Intensiva, Hospital Universitari Sant Pau, Barcelona, Spain.

${ }^{10} \mathrm{CHU}$ de Poitiers, Médecine Intensive Réanimation, Poitiers, France.

${ }^{11}$ INSERM, CIC-1402, équipe ALIVE, Poitiers, France.

${ }^{12}$ Université de Poitiers, Faculté de Médecine et de Pharmacie de Poitiers, Poitiers, France.

${ }^{13}$ Department of Intensive Care Medicine, Deenanath Mangeshkar Hospital and Research Centre, Pune, India.

${ }^{14}$ Intensive Care Unit, Hospital Infanta Sofia, Madrid, Spain.

${ }^{15}$ Department of Medical, Oral and Biotechnological Sciences, Gabriele d'Annunzio University of Chieti-Pescara, AND Department of Anesthesiology and Critical Care, SS. Annunziata Hospital, Chieti, Italy.

${ }^{16}$ Department of Epidemiology and Preventive Medicine, Monash University, Melbourne, VIC, Australia.

${ }^{17}$ University Hospital of Montpellier and Saint Eloi Hospital, Montpellier University, Montpellier, France.

${ }^{18}$ Interdepartmental Division of Critical Care Medicine, University of Toronto, Toronto, ON, Canada.

${ }^{19}$ Department of Health Research Methods, Evidence and Impact, McMaster University, Hamilton, ON, Canada.

Copyright (C) 2020 by the Society of Critical Care Medicine and Wolters Kluwer Health, Inc. All Rights Reserved.
Ohjective: The role of high-flow nasal cannula during and before intubation is unclear despite a number of randomized clinical trials. Our objective was to conduct a systematic review and metaanalysis examining the benefits of high-flow nasal cannula in the peri-intubation period.

Data Sources: We performed a comprehensive search of relevant databases (MEDLINE, EMBASE, and Web of Science).

Study Selection: We included randomized clinical trials that compared high-flow nasal cannula to other noninvasive oxygen delivery systems in the peri-intubation period.

Data Extraction: Our primary outcome was severe desaturation (defined as peripheral oxygen saturation reading $<80 \%$ during intubation). Secondary outcomes included peri-intubation complications, apneic time, $\mathrm{PaO}_{2}$ before and after intubation, $\mathrm{PaCO}_{2}$ after intubation, ICU length of stay, and short-term mortality.

Data Synthesis: We included 10 randomized clinical trials ( $n=1,017$ patients). High-flow nasal cannula had no effect on the occurrence rate of peri-intubation hypoxemia (relative risk, $0.98 ; 95 \% \mathrm{Cl}, 0.68-1.42 ; 0.3 \%$ absolute risk reduction, moderate certainty), serious complications (relative risk, 0.87; 95\% $\mathrm{Cl}, 0.71-1.06$ ), apneic time (mean difference, 10.3s higher with high-flow nasal cannula; $95 \% \mathrm{Cl}, 11.0 \mathrm{~s}$ lower to $31.7 \mathrm{~s}$ higher), $\mathrm{PaO}_{2}$ measured after preoxygenation (mean difference, $3.6 \mathrm{~mm} \mathrm{Hg}$ higher; $95 \% \mathrm{Cl}, 3.5 \mathrm{~mm} \mathrm{Hg}$ lower to $10.7 \mathrm{~mm} \mathrm{Hg}$ higher), or $\mathrm{PaO}_{2}$ measured after intubation (mean difference, $27.0 \mathrm{~mm} \mathrm{Hg}$ higher; $95 \% \mathrm{Cl}, 13.2 \mathrm{~mm} \mathrm{Hg}$ lower to $67.2 \mathrm{~mm} \mathrm{Hg}$ higher), when compared with conventional oxygen therapy. There was also no effect on postintubation $\mathrm{PaCO}_{2}, \mathrm{ICU}$ length of stay, or 28-day mortality.

Conclusions: We found moderate-to-low certainty evidence that the use of high-flow nasal cannula likely has no effect on severe desaturation, serious complications, apneic time, oxygenation, ICU length of stay, or overall survival when used in the peri-intu- 
bation period when compared with conventional oxygen therapy.

Key Words: artificial respiration; hypoxia; intubation; noninvasive ventilation; oxygen inhalation therapy; systematic review

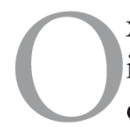
xygen delivery via a high-flow nasal cannula (HFNC) is increasingly being used as an alternative to standard oxygen therapy or noninvasive ventilation (NIV) for patients with hypoxemic respiratory failure $(1,2)$. HFNC allows for delivery of high rates of humidified and heated oxygen (up to $70 \mathrm{~L}$ / min) (1). This method of oxygen delivery also creates a modest amount of positive end-expiratory pressure, enables carbon dioxide $\left(\mathrm{CO}_{2}\right)$ washout, and decreases nasopharyngeal resistance ( 3 , 4). Furthermore, it is hypothesized that high-flow therapy results in washout of the upper airways because of the increased airway pressures it generates. As a result, the patient rebreathes less expired air from the upper airways and more fresh air is delivered by the nasal high flow $(5,6)$. When compared with other oxygen delivery devices, HFNC may be more comfortable and less obtrusive (4). Consequently, HFNC may confer benefit by preventing hypoxemia and increasing apnea time prior to intubation (4). In fact, a recent narrative review on HFNC use presents it as a reasonable option for preoxygenation in the peri-intubation period (7).

The largest randomized clinical trial comparing HFNC to other modes of NIV in the peri-intubation period was published in March 2019 and showed no difference in clinical outcomes related to the use of alternative oxygen delivery devices (8). We conducted a systematic review and meta-analysis of randomized controlled trials (RCTs) examining the effects of HFNC compared with bag mask or NIV in the peri-intubation period.

\section{MATERIALS AND METHODS}

We registered the protocol for this systematic review and metaanalysis on International prospective register of systematic reviews (\# 42019120959) and used the Preferred Reporting Items for Systematic Reviews and Meta-Analysis checklist to report our findings (Supplementary Table 4, Supplemental Digital Content 1, http://links.lww.com/CCM/F294).

\section{Data Sources and Searches}

We performed a comprehensive search of all relevant databases from January 1, 2007, to October 25, 2018, recognizing that HFNC was not widely used before 2007. Our search strategy used key words including "human" OR "adult" OR "mature" or "grown" AND "high flow nasal cannula" OR "high flow nasal therapy" OR "high flow nasal oxygen" OR "high flow oxygen therapy" OR "high flow therapy" OR "optiflow (respiration)" OR "nasal highflow." We did not restrict studies to language or quality. An updated search was conducted on April 15, 2019, and on November 6, 2019.

\section{Study Selection}

Two reviewers screened citations independently and in duplicate (D.G., D.W.) from our initial search in two stages; first we screened the title and abstract and then, we screened the full text of potentially relevant abstracts. We captured reasons for study exclusion at the full-text review stage. A third author (B.R.) adjudicated disagreements.

We included RCTs that compared HFNC to other noninvasive oxygen delivery modalities in the peri-intubation period. We defined peri-intubation period as the time between induction of anesthesia or administration of sedative agents and until connection of the patient to the mechanical ventilator. We included trials with nonhypoxemic patients (e.g., perioperative) and those with hypoxemic patients (e.g., critically ill patients). We excluded case reports, case series, and observational studies. Our primary outcome was severe desaturation (uniformly defined as peripheral capillary oxygen saturation $\left[\mathrm{SpO}_{2}\right]<80 \%$ ). Secondary outcomes included peri-intubation complications, apneic time, $\mathrm{PaO}_{2}$ (post preoxygenation, postintubation), post intubation $\mathrm{PaCO}_{2}$, ICU length of stay (LOS), and short-term mortality. We defined post preoxygenation $\mathrm{PaO}_{2}$ as the first $\mathrm{PaO}_{2}$ from the first arterial blood gas (ABG) taken after the defined period of preoxygenation was completed and we defined postintubation $\mathrm{PaO}_{2}$ as the first $\mathrm{PaO}_{2}$ from the first ABG after intubation. The above definitions held for postintubation $\mathrm{PaCO}_{2}$ as well.

\section{Data Extraction and Quality Assessment}

Two reviewers (D.C., D.G.) abstracted data independently and in duplicate using a structured data abstraction form. A third reviewer resolved any disagreements. We collected study characteristics, demographic data, details and definitions of the intervention and control, and details and definitions of the predefined study outcomes. If only $\mathrm{SpO}_{2}$ was reported and not $\mathrm{PaO}_{2}$, we converted to $\mathrm{PaO}_{2}$ using the equations derived by Severinghaus (9).

We assessed risk of bias ( $\mathrm{ROB}$ ) using a modified Cochrane ROB tool for RCTs (10) and assessed for randomization sequence generation, allocation concealment, blinding, incomplete data, selective reporting, and other bias. ROB was assessed independently and in duplicate. We rated the ROB for each domain as "low," "probably low," "high," or "probably high." We rated the overall ROB as the highest risk attributed to any criterion, except for blinding. Because the nature of the intervention in these trials made blinding virtually impossible, we rated the overall $\mathrm{ROB}$ to be low for trials that had only had issues with blinding of the intervention. We assessed the overall certainty of evidence regarding each outcome using the Grading of Recommendations, Assessment, Development, and Evaluation (GRADE) framework (11).

\section{Data Analysis}

We used the DerSimonian-Laird random-effects model with inverse-variance weighting to generate pooled treatment effect across trials. We measured heterogeneity between trials using the Chi-square test (with a $p$ value $<0.10$ denoting significant heterogeneity), the $P^{2}$ statistic (with a value $>50 \%$ denoting substantial heterogeneity), and visual inspection of the forest plots (12). We present results as relative risk (RR) for dichotomous outcomes and mean difference (MD) for continuous outcomes with 95\% continuous intervals. We performed all 
statistical analyses using RevMan 5.3 (Cochrane Collaboration, Oxford, United Kingdom) software.

We planned three a priori subgroup analyses comparing trials evaluating: 1) operative patients versus critically ill patients with hypoxemic respiratory failure (hypothesizing that patients with hypoxemic respiratory failure would benefit more with HFNC); 2) any form of NIV as a comparator versus facemask and bag mask as comparator (hypothesizing that patients undergoing ventilation with facemask/bag mask would benefit more from HFNC); and 3) high ROB trials versus low ROB trials (hypothesizing that trials with a high ROB would show a greater effect of HFNC compared with trials with low ROB). We also performed sensitivity analyses for the outcomes of post preoxygenation $\mathrm{PaO}_{2}$ and postintubation $\mathrm{PaO}_{2}$ excluding trials that reported only $\mathrm{SpO}_{2}(8,13-15)$.

We conducted trial sequential analysis (16) using randomeffect model for the peri-intubation hypoxemia outcome. We used a statistical significance level of $5 \%$, a power of $80 \%$, and a $\mathrm{RR}$ reduction of $15 \%$. We used a model variance-based heterogeneity correction. Trial sequential analysis was performed using Trial Sequential Analysis version 0.9 .5 .10 beta (Copenhagen Trial Unit, Centre for Clinical Intervention Research, Rigshospitalet, Copenhagen, Denmark, www.ctu.dk/tsa).

\section{RESULTS}

\section{Search Strategy and Study Characteristics}

We reviewed 649 citations and ultimately included 10 RCTs with a total of 1,017 patients $(4,8,13-15,17-21)$ (Fig. 1). We considered, but ultimately excluded, an 11th trial that examined the use of standard nasal cannula for peri-intubation oxygenation (22). Although this trial labeled the intervention "high flow," standard soft plastic nasal cannula were used with flows of $15 \mathrm{~L} / \mathrm{min}$ of oxygen rather than specialized nasal cannula (e.g., Optiflow system) and average flows employed were significantly less than those of other included trials $(15 \mathrm{~L} / \mathrm{min}$ here as compared with $50-70 \mathrm{~L} / \mathrm{min}$ in the other high-flow trials) (23).

The characteristics of included trials are shown in Supplementary Table 3 (Supplemental Digital Content 1, http:// links.lww.com/CCM/F294). The trials included had randomized between 33 and 333 patients. Five of the 10 trials examined nonhypoxemic patients undergoing intubation during induction of general anesthesia before surgery $(14,17,19-21)$, whereas the other five trials examined critically ill hypoxemic patients (4, $8,13,15,18)$. Of the preoperative trials, two examined patients undergoing emergency surgery $(14,20)$, one trial evaluated patients undergoing bariatric surgery (17), one evaluated obese patients undergoing any type of surgery (21), and one examined patients undergoing neurosurgery (19). All the perioperative trials excluded patients with underlying respiratory conditions. All included trials followed a standardized approach during intubation. Once patients were randomized, they were preoxygenated using either the intervention or control device for a set period of time, ranging between 3 and 7 minutes. Once this was completed, patients were sedated, paralyzed, and then intubated. In the intervention arm of all trials, the HFNC was left in place while intubating patients. Successful intubation was confirmed by end tidal $\mathrm{CO}_{2}$ and was followed by connection to the mechanical ventilator.

Of the trials examining critically ill patients, all except one (15) included patients with hypoxemic respiratory failure. Definitions for hypoxemic respiratory failure varied but all required that patients have a $\mathrm{PaO}_{2} / \mathrm{FIO}_{2}$ ratio below 300, along with some combination of an elevated respiratory rate and decreased $\mathrm{SpO}_{2}$. One trial (15) examined only patients with mild or moderate hypoxemia and excluded patients with $\mathrm{PaO}_{2} / \mathrm{FIO}_{2}$ less than 200. Sensitivity analysis performed excluding this trial showed no difference of significance in any of the outcomes.

Two of the 10 trials compared $\operatorname{HFNC}$ to $\operatorname{NIV}(8,21)$, whereas six compared HFNC to facemask or bag mask (4, $13-15,19,20)$. One threearmed trial compared HFNC to NIV and to facemask with bag mask (17). Finally, one

\section{Not peri-intubation (n=88) -Not RCT ( $n=69$ ) \\ Did not report outcome \\ of interest $(n=7)$ - Did not use Optiflow system ( $n=2)$}

Figure 1. Preferred Reporting Items for Systematic Reviews and Meta-Analysis flow diagram-study selection. $\mathrm{RCT}=$ randomized clinical trial 
trial compared HFNC with NIV to NIV alone (18). The intervention of choice in all the trials were the high-flow heated and humidified nasal oxygen (Optiflow; Fisher and Paykel Healthcare, Auckland, New Zealand) device set to a $\mathrm{FIO}_{2}$ of 1.0 and gas flow rates ranging between 50 and $70 \mathrm{~L} / \mathrm{min}$.

Supplementary Table 1 (Supplemental Digital Content 1, http://links.lww.com/CCM/F294) summarizes the individual trial ROB. None of the trials blinded patients or clinicians. All trials except one (20) were judged to be at low or probably low risk of other forms of bias.

\section{Outcomes}

Supplementary Table 2 (Supplemental Digital Content 1, http://links.lww.com/CCM/F294) shows pooled estimates and GRADE certainties.

\section{Primary Outcome}

Compared with conventional oxygen therapy (i.e., face mask and/or bag mask), HFNC demonstrated no effect on peri-intubation hypoxemia (RR, 0.98 ; 95\% CI, 0.68-1.42; 0.3\% absolute risk reduction; $95 \% \mathrm{CI}, 4.9 \%$ reduction to $6.5 \%$ increase, moderate certainty, Fig. 2). All trials defined peri-intubation hypoxia as $\mathrm{SpO}_{2}$ less than $80 \%$ in the period between induction of anesthetic medication and/or commencement of laryngoscopy to connection to the mechanical ventilator. No credible subgroup effects were found on peri-intubation hypoxemia based on patient type (ICU vs preoperative patients, Fig. 2), the comparator used (NIV vs face mask/bag mask [Supplementary Fig. 1, Supplemental Digital Content 2, http://links. lww.com/CCM/F295; legend, Supplemental Digital Content 1, http://links.lww.com/CCM/F294]), or individual study ROB (high risk vs low risk [Supplementary Fig. 2, Supplemental Digital Content 3, http://links.lww.com/CCM/F296; legend,
Supplemental Digital Content 1, http://links.lww.com/CCM/ F294]). The trial sequential analysis for this outcome showed that the required information size for this outcome was not met (Supplementary Fig. 3, Supplemental Digital Content 4, http://links.lww.com/CCM/F297; legend, Supplemental Digital Content 1, http://links.lww.com/CCM/F294).

\section{Secondary Outcomes}

Similarly, no difference was found in the composite outcome of serious peri-intubation complications with HFNC compared with conventional oxygen therapy (RR, 0.87; 95\% CI, 0.71$1.06 ; 6.2 \%$ absolute risk reduction; $95 \% \mathrm{CI}, 13.9 \%$ reduction to $2.9 \%$ increase, low certainty, Fig. 3). Of note, peri-intubation complications were defined differently across studies (Supplementary Table 3, Supplemental Digital Content 1, http://links. lww.com/CCM/F294). All studies reported severe hypoxemia ( $\mathrm{SpO}_{2}$ below $80 \%$ ) and severe hypotension. However, hypotension was defined differently across studies with some studies defining it as SBP less than $80 \mathrm{~mm} \mathrm{Hg}$, and some defining it as systolic blood pressure (SBP) less than $90 \mathrm{~mm} \mathrm{Hg}$.

When comparing HFNC to conventional oxygen therapy, no effect was found on total apneic time (MD, 10.3 s longer; 95\% CI, 11.0 s shorter to 31.7 s longer, low certainty; Fig. 4). There was also no effect on $\mathrm{PaO}_{2}$ after preoxygenation (MD, $3.6 \mathrm{~mm} \mathrm{Hg}$ higher; 95\% CI, $3.5 \mathrm{~mm} \mathrm{Hg}$ lower to $10.7 \mathrm{~mm}$ $\mathrm{Hg}$ higher, moderate certainty; Fig. 5) or $\mathrm{PaO}_{2}$ after intubation (MD, $27.0 \mathrm{~mm} \mathrm{Hg}$ higher; 95\% CI, $13.2 \mathrm{~mm} \mathrm{Hg}$ lower to $67.2 \mathrm{~mm}$ Hg higher, moderate certainty; Supplementary Fig. 4, Supplemental Digital Content 5, http://links.lww.com/CCM/ F298; legend, Supplemental Digital Content 1, http://links.lww. com/CCM/F294) with HFNC. Furthermore, there was no effect of HFNC when compared with conventional oxygen therapy in any of the other secondary outcomes including postintubation

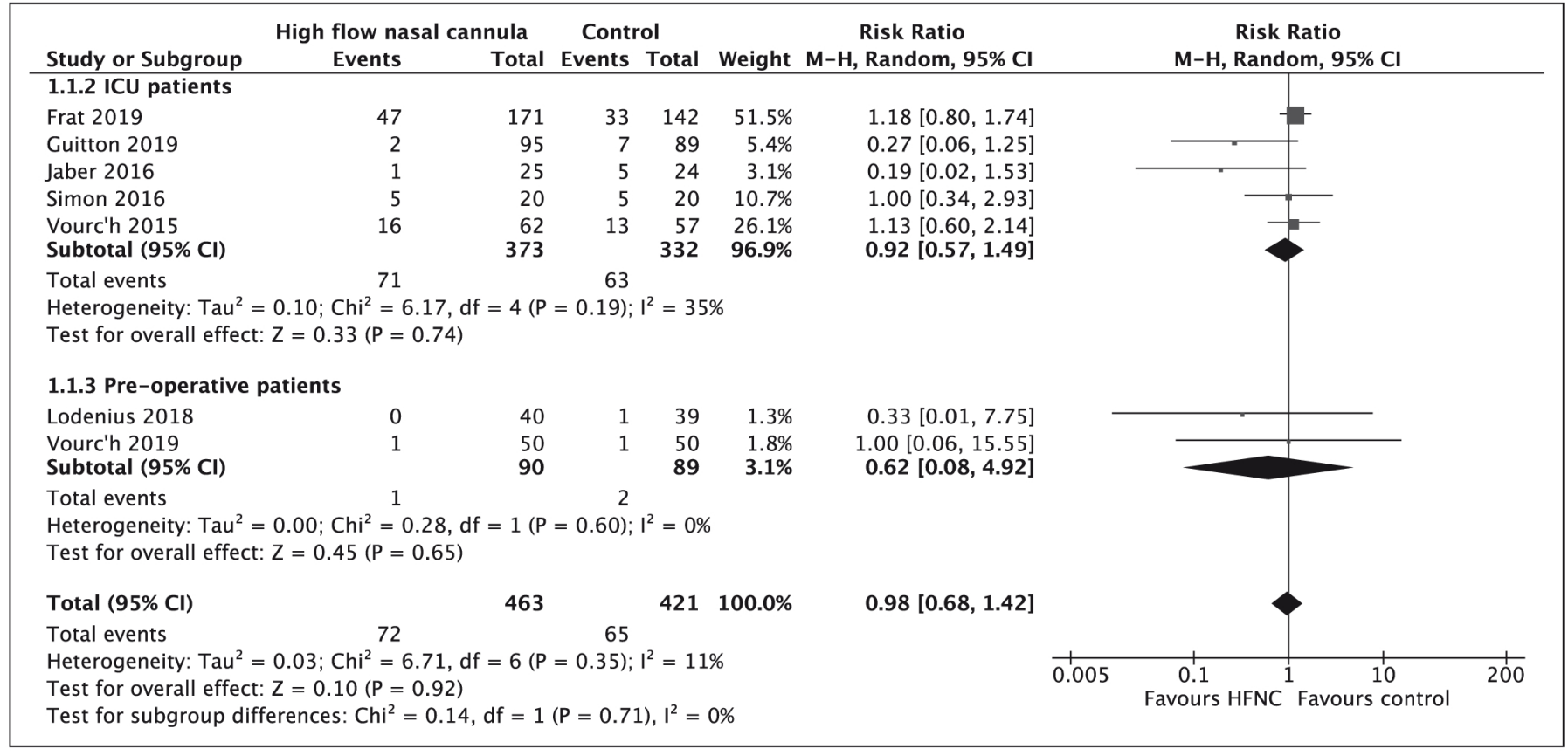

Figure 2. Peri-intubation hypoxemia forest plot. $d f=$ degrees of freedom, $\mathrm{M}-\mathrm{H}=$ Mantel-Haenszel. 


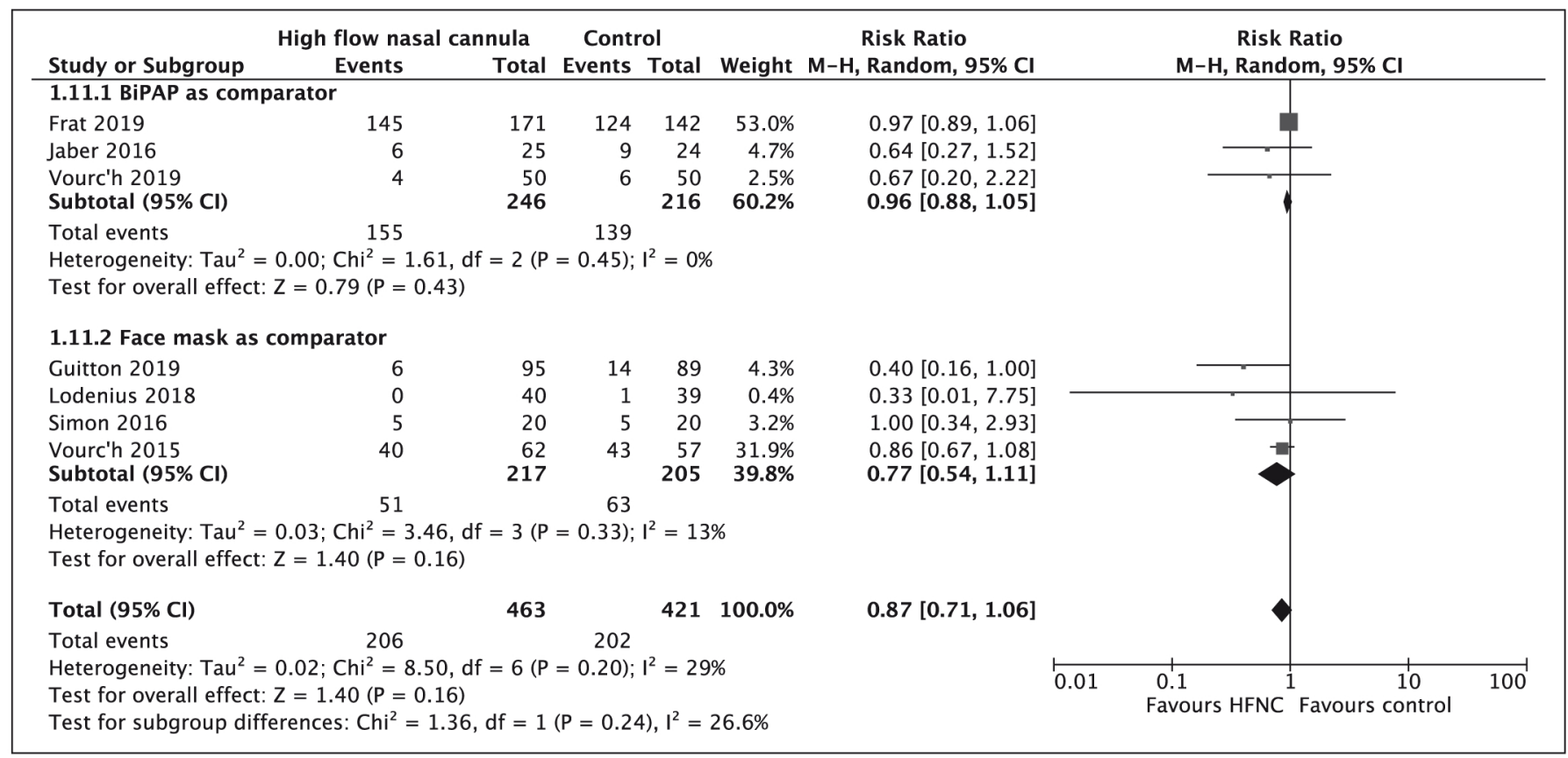

Figure 3. Serious peri-intubation complications forest plot. BiPAP $=$ bilevel positive airway pressure, $d f=$ degrees of freedom, $\mathrm{HFNC}=$ high-flow nasal cannula

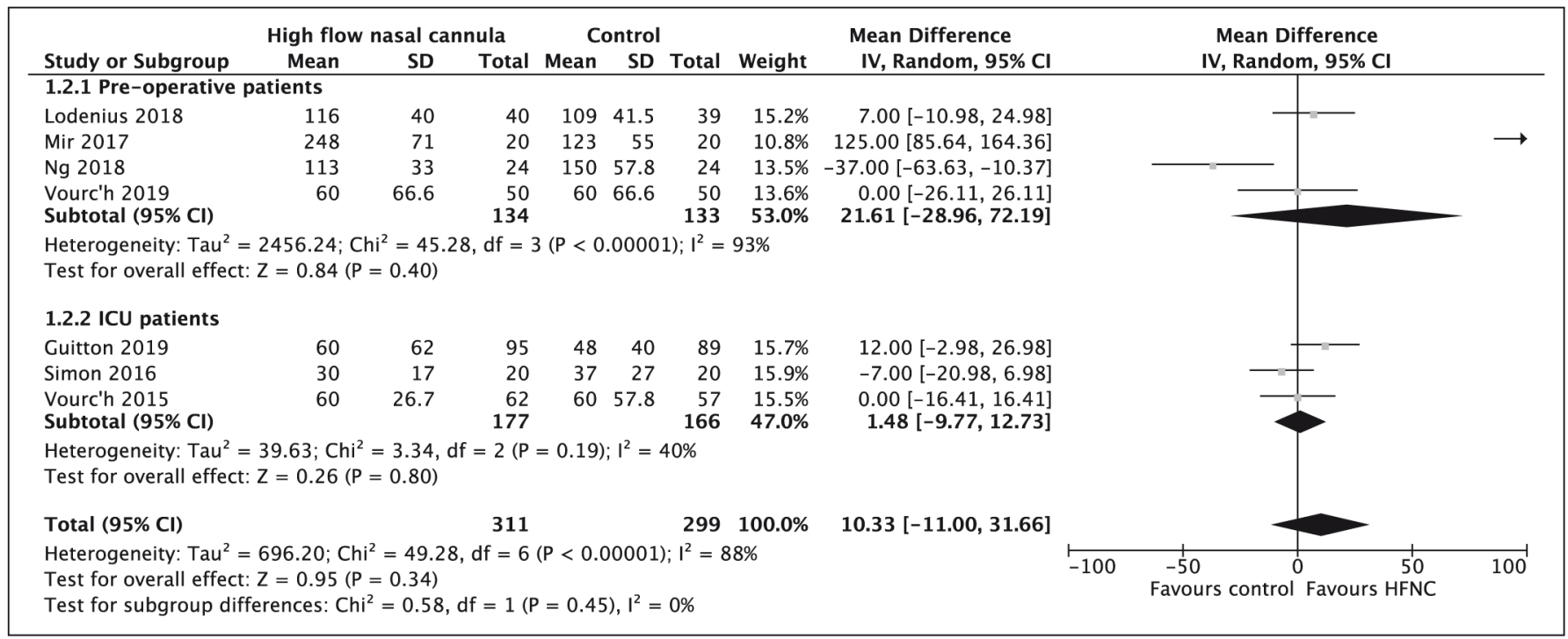

Figure 4. Total apneic time in seconds forest plot subdivided by patient subtype. $d f=$ degrees of freedom, HFNC $=$ high-flow nasal cannula.

$\mathrm{PaCO}_{2}$, ICU LOS, and 28-day mortality (Supplementary Fig. 5, Supplemental Digital Content 6, http://links.lww.com/CCM/ F299; Supplementary Fig. 6, Supplemental Digital Content 7, http://links.lww.com/CCM/F300; and Supplementary Fig. 7, Supplemental Digital Content 8, http://links.lww.com/CCM/ F301; legend, Supplemental Digital Content 1, http://links. lww.com/CCM/F294).

\section{Subgroup and Sensitivity Analyses}

Further subgroup analyses based on patient or comparator subtype showed that there was a significant increase in $\mathrm{PaO}_{2}$ levels after preoxygenation in the operative subgroup of patients who received HFNC (Fig. 4). There was no credible effect on any of the other outcomes of interest (Supplementary
Fig. 4, Supplemental Digital Content 5, http://links.lww.com/ CCM/F298; Supplementary Fig. 5, Supplemental Digital Content 6, http://links.lww.com/CCM/F299; Supplementary Fig. 6, Supplemental Digital Content 7, http://links.lww.com/CCM/ F300; Supplementary Fig. 7, Supplemental Digital Content 8, http://links.lww.com/CCM/F301; Supplementary Fig. 8, Supplemental Digital Content 9, http://links.lww.com/CCM/F302; and Supplementary Fig. 9, Supplemental Digital Content 10, http://links.lww.com/CCM/F303; legend, Supplemental Digital Content 1, http://links.lww.com/CCM/F294).

Sensitivity analysis excluding trials that reported only $\mathrm{SpO}_{2}$ did not alter the conclusions (Supplementary Fig. 10, Supplemental Digital Content 11, http://links.lww.com/ CCM/F304; and Supplementary Fig. 11, Supplemental 


\begin{tabular}{|c|c|c|c|c|c|c|c|c|c|c|c|c|}
\hline \multirow{2}{*}{$\begin{array}{l}\text { Study or Subgroup } \\
\text { 1.5.1 Pre-operative patier }\end{array}$} & \multicolumn{3}{|c|}{ High flow nasal cannula } & \multicolumn{3}{|c|}{ Control } & \multirow[t]{2}{*}{ Weight } & \multirow[t]{2}{*}{$\begin{array}{l}\text { Mean Difference } \\
\text { IV, Random, } 95 \% \mathrm{CI}\end{array}$} & \multicolumn{4}{|c|}{$\begin{array}{l}\text { Mean Difference } \\
\text { IV, Random, } 95 \% \mathrm{CI}\end{array}$} \\
\hline & & & & & & & & & & & & \\
\hline Heinrich BiPAP 2014 & 405 & 99 & 6 & 373 & 88 & 11 & $0.5 \%$ & $32.00[-62.76,126.76]$ & & & & \\
\hline Heinrich Facemask 2014 & 405 & 99 & 5 & 315 & 62 & 11 & $0.6 \%$ & $90.00[-4.19,184.19]$ & & & & \\
\hline $\mathrm{Ng} 2018$ & 471 & 64 & 24 & 357 & 93 & 24 & $2.3 \%$ & $114.00[68.83,159.17]$ & & & & \\
\hline Subtotal $(95 \% \mathrm{Cl})$ & & & 35 & & & 46 & $3.4 \%$ & $93.68[49.81,137.55]$ & & & & \\
\hline \multicolumn{13}{|c|}{$\begin{array}{l}\text { Heterogeneity: } \mathrm{Tau}^{2}=282.88 ; \mathrm{Chi}^{2}=2.37, \mathrm{df}=2(\mathrm{P}=0.31) ; \mathrm{I}^{2}=16 \% \\
\text { Test for overall effect: } Z=4.19(P<0.0001)\end{array}$} \\
\hline \multicolumn{13}{|l|}{ 1.5.2 ICU patients } \\
\hline Frat 2019 & 82 & 6 & 171 & 91 & 5 & 142 & $31.5 \%$ & $-9.00[-10.22,-7.78]$ & & - & & \\
\hline Guitton 2019 & 167 & 0.001 & 95 & 167 & 0.001 & 89 & $31.8 \%$ & $0.00[-0.00,0.00]$ & & & 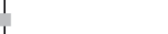 & \\
\hline Jaber 2016 & 171 & 181 & 25 & 100 & 104 & 24 & $0.7 \%$ & $71.00[-11.25,153.25]$ & & & & \\
\hline Simon 2016 & 184 & 107 & 20 & 173 & 86 & 20 & $1.3 \%$ & $11.00[-49.16,71.16]$ & & & & \\
\hline Vourc'h 2015 & 92 & 5 & 62 & 84 & 5 & 57 & $31.2 \%$ & $8.00[6.20,9.80]$ & & & 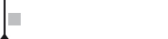 & \\
\hline Subtotal $(95 \% \mathrm{Cl})$ & & & 373 & & & 332 & $96.6 \%$ & $0.27[-6.64,7.19]$ & & & 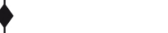 & \\
\hline \multicolumn{13}{|c|}{$\begin{array}{l}\text { Heterogeneity: } \mathrm{Tau}^{2}=37.68 ; \mathrm{Chi}^{2}=288.54, \mathrm{df}=4(\mathrm{P}<0.00001) ; \mathrm{I}^{2}=99 \% \\
\text { Test for overall effect: } Z=0.08(P=0.94)\end{array}$} \\
\hline Total $(95 \% \mathrm{Cl})$ & & & 408 & & & 378 & $100.0 \%$ & $3.59[-3.49,10.68]$ & & & 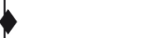 & \\
\hline \multicolumn{7}{|c|}{$\begin{array}{l}\text { Heterogeneity: } \mathrm{Tau}^{2}=41.01 ; \mathrm{Chi}^{2}=316.96, \mathrm{df}=7(\mathrm{P}<0.00001) ; \mathrm{I}^{2}=98 \% \\
\text { Test for overall effect: } \mathrm{Z}=0.99(\mathrm{P}=0.32) \\
\text { Test for subgroup differences: } \mathrm{Chi}^{2}=16.99, \mathrm{df}=1(\mathrm{P}<0.0001), \mathrm{I}^{2}=94.1 \%\end{array}$} & & & -200 & $\begin{array}{ll}1 & +100 \\
\text { Favours control }\end{array}$ & $\begin{array}{lr} \\
0 & 100 \\
\end{array}$ & 200 \\
\hline
\end{tabular}

Figure 5. $\mathrm{PaO}_{2}$ after preoxygenation forest plot subdivided by patient subtype. $d f=$ degrees of freedom, $\mathrm{HFNC}=$ high-flow nasal cannula.

Digital Content 12, http://links.lww.com/CCM/F305; legend, Supplemental Digital Content 1, http://links.lww.com/CCM/ F294).

\section{DISCUSSION}

This systematic review and meta-analysis demonstrate that the use of HFNC in the peri-intubation period likely provides no benefit compared with alternative oxygen delivery devices with regard to physiologically and clinically important outcomes. Further, subgroup analysis based on patient subtype, comparator, and individual study ROB did not identify any specific populations or groups that would require alternative consideration. Of note, most events, specifically for the outcomes of serious complications, hypoxemic events, and deaths, were reported in trials that evaluated critically ill patients. The overall certainty of evidence was moderate to low and was limited primarily by imprecision.

Although other reviews have examined the role $\operatorname{HFNC}(2,22$, 24-26) in various clinical scenarios, only one other review has looked at HFNC use in the peri-intubation period (27). However, although Cabrini et al (27) looked at all RCTs that examined any drug, technique or device that could improve tracheal intubation, this review was the first one to focus specifically on HFNC. Furthermore, our review includes data from three very recently published RCTs, one of which is the largest trial included in this systematic review $(8,15)$, making this also the most up-to-date review on the topic. Our comprehensive search strategy, preregistered methodology, and assessment of certainty using the GRADE approach are also strengths of this study.

Our review also has limitations. First, the trials included varied in the patients enrolled and the comparators used. However, this clinical heterogeneity did not also manifest as a high degree of statistical heterogeneity. Furthermore, we found no evidence to support credible subgroup differences when we examined these variables. Second, one secondary outcome (peri-intubation complications) was comprised of several components with variable clinical importance. In addition, trials did not consistently report $\mathrm{SpO}_{2}$ or $\mathrm{PaO}_{2}$ levels when measuring hypoxia. As a result, for trials that reported only $\mathrm{SpO}_{2}$ levels, we had to use a conversion equation to convert those levels to $\mathrm{PaO}_{2}$. Because this equation is not always reliable and varies based on $\mathrm{pH}$, this may have introduced a degree of error in our $\mathrm{PaO}_{2}$-dependent outcomes. However, when we performed a sensitivity analysis excluding these studies, our results did not change. Finally, all trials included in this review were unblinded.

Given that no demonstrable benefit was found in the outcomes measured with the use of HFNC peri-intubation, it may be claimed that it confers no advantage in this clinical situation. Our primary hypothesis for lack of HFNC superiority was that most patients, even in the critically ill subgroup, likely had enough functional residual capacity even with conventional oxygen preoxygenation to prevent severe desaturation and related complications during the intubation period. Mean apnea times were less than 2 minutes for all studies included except for one study in the preoperative group that had a mean apnea time of 4 minutes in the HFNC arm (14). In the critically ill patient population, who are at much higher risk of hypoxia, the mean apnea times were on average less than a minute. Because HFNC (delivered through the nostrils) allows for apneic oxygenation in contrast to face mask therapy, it likely has a beneficial effect during prolonged apneic periods or very severe hypoxia. As a result, we hypothesize that the benefits of HFNC may only be seen during prolonged intubations or patients with very severe hypoxia (partial pressure of arterial oxygen/fraction of inhaled oxygen ratio $<100$ ), subgroups that we were unable to isolate in our included studies.

However, our study is only as good as the data reported. The HFNC may be better tolerated by selected patients, especially those more awake or claustrophobic. These benefits could contribute to patient comfort and or facilitate intubation for clinicians when used during intubation. Only one of the included 
trials, reported on the number of intubation attempts, type of device used for intubation, overall hypoxemic time, or the quality of airway visualization (21). Studies have also shown that such emergency airway situations are accompanied by considerable physician stress. This too was not measured. Some authors have suggested that apneic time may be a surrogate for operator difficulty during intubation (28). Although this review did not demonstrate a difference in apneic time, only seven of the 10 trials reported this outcome. Notwithstanding, variance in apneic time has also been reported in patients who are considered to be easy or moderately difficult intubations (28). These findings may be influenced by other factors. For example, operators may take their time with patients who are easier to intubate or allow less-experienced operators attempt intubation first recognizing that selected patients may have greater reserve. Further research on many of these aspects is still required.

\section{CONCLUSIONS}

The studies published to date suggest with low-to-moderate certainty evidence that the use of HFNC has no measurable effect on hypoxemia, serious complications, oxygenation, ICU LOS, or overall survival when used in the peri-intubation period. These findings do not support routine use of HFNC during endotracheal intubation of critically ill adults. However, further studies on this topic will be needed to answer this question with higher certainty. Furthermore, questions regarding performance of HFNC for ease of intubation procedure, operator stress level, difficult intubations, and severe hypoxia remain to be addressed.

Dr. Mauri received speaking fees from Fisher and Paykel unrelated to the present work. Dr. Mancebo received funding from Faron, Medtronic, and IMT medical (travel support); personal fees from Faron and Medtronic; and he disclosed that Fisher Paykel and A-Lung provided medical equipment to participate in multicenter clinical trials (high-flow nasal oxygen therapy and extracorporeal carbon dioxide removal, respectively), and Covidien (Medtronic) and Canadian Institutes of Health Research sponsored a multicenter randomized clinical trial. IMT Medical provided travel and hotel expenses to attend a meeting. Dr. Maggiore disclosed that he is the principal investigator of the Impact of Nasal High-flow vs Venturi Mask Oxygen Therapy on Weaning Outcome: A Multicenter, Randomized, Controlled Trial (RINO trial) (Clinicaltrials.gov, NCT02107183), which was supported by Fisher and Paykel Healthcare, and he received funding from lecturing for General Electric Healthcare and Draeger Medical. Dr. Brochard's institution received funding from Medtronic Covidien and Fisher and Paykel. Dr. Rochwerg is supported by a Hamilton Health Sciences Early Career Research Award. The remaining authors have disclosed that they do not have any potential conflicts of interest.

Address requests for reprints to: Bram Rochwerg, MD, Division of Critical Care, Department of Medicine, Juravinski Hospital, 711 Concession St, Hamilton, ON, Canada L8V 1C3. E-mail: rochwerg@mcmaster.ca

\section{REFERENCES}

1. Lee JH, Rehder KJ, Williford L, et al: Use of high flow nasal cannula in critically ill infants, children, and adults: A critical review of the literature. [Internet]. Intensive Care Med 2013; 39:247-57

2. Rochwerg B, Granton D, Wang DX, et al: High-flow nasal cannula compared with conventional oxygen therapy for acute hypoxemic respiratory failure: author's reply. Intensive Care Med 2019; 45:1171
3. Cortegiani A, Accurso G, Mercadante S, et al: High flow nasal therapy in perioperative medicine: From operating room to general ward. BMC Anesthesio/ 2018; 18:166

4. Simon M, Wachs $C$, Braune $S$, et al: High-flow nasal cannula versus bag-valve-mask for preoxygenation before intubation in subjects with hypoxemic respiratory failure. Respir Care 2016; 61:1160-1167

5. Goligher EC, Slutsky AS: Not just oxygen? mechanisms of benefit from high-flow nasal cannula in hypoxemic respiratory failure. Am J Respir Crit Care Med 2017; 195:1128-1131

6. Möller W, Feng S, Domanski U, et al: Nasal high flow reduces dead space. J Appl Physiol (1985) 2017; 122:191-197

7. Drake MG: High-flow nasal cannula oxygen in adults: an evidence-based assessment. Ann Am Thorac Soc 2018; 15:145-155

8. Frat JP, Ricard JD, Quenot JP, et al: Non-invasive ventilation versus high-flow nasal cannula oxygen therapy with apnoeic oxygenation for preoxygenation before intubation of patients with acute hypoxaemic respiratory failure: A randomised, multicentre, open-label trial [Internet]. Lancet Respir Med 2019; 7:303-312

9. Severinghaus JW: Simple, accurate equations for human blood $\mathrm{O} 2$ dissociation computations. J Appl Physiol Respir Environ Exerc Physiol 1979; 46:599-602

10. Higgins JP, Altman DG, Gøtzsche PC, et al; Cochrane Bias Methods Group; Cochrane Statistical Methods Group: The Cochrane Collaboration's tool for assessing risk of bias in randomised trials. BMJ 2011; 343:d5928

11. Guyatt GH, Oxman AD, Vist GE, et al; GRADE Working Group: GRADE: An emerging consensus on rating quality of evidence and strength of recommendations. BMJ 2008; 336:924-926

12. Deeks J, Higgins J: Statistical Algorithms in Review Manager 5. Statistical Methods Group of the Cochrane Collaboration - Google Scholar [Internet]. Stat Methods Groups Cochrane Collab 2010. Cited July 8, 2017. Available at: https://scholar-google-ca.libaccess.lib. mcmaster.ca/scholar?hl=en\&q=Statistical+Algorithms+in+Review+M anager+5.+Statistical+Methods+Group+of+the+Cochrane+Collabor ation\&btnG=\&as_sdt=1\%2C5\&as_sdtp=. Accessed July 15, 2019

13. Vourc'h M, Asfar P, Volteau $C$, et al: High-flow nasal cannula oxygen during endotracheal intubation in hypoxemic patients: A randomized controlled clinical trial. Intensive Care Med 2015; 41: 1538-1548

14. Mir F, Patel A, lqbal $R$, et al: A randomised controlled trial comparing transnasal humidified rapid insufflation ventilatory exchange (THRIVE) pre-oxygenation with facemask pre-oxygenation in patients undergoing rapid sequence induction of anaesthesia. Anaesthesia 2017; 72:439-443

15. Guitton C, Ehrmann S, Volteau C, et al: Nasal high-flow preoxygenation for endotracheal intubation in the critically ill patient: $A$ randomized clinical trial. Intensive Care Med 2019; 45:447-458

16. Wetterslev J, Jakobsen JC, Gluud C: Trial Sequential Analysis in systematic reviews with meta-analysis. BMC Med Res Methodol 2017; $17: 39$

17. Sebastian $H$, Thomas $H$, Benedikt $S$, et al: Benefits of heated and humidified high flow nasal oxygen for preoxygenation in morbidly obese patients undergoing bariatric surgery: A Randomized Controlled Study. J Obes Bariatrics 2014; 1:1-7

18. Jaber S, Monnin M, Girard M, et al: Apnoeic oxygenation via high-flow nasal cannula oxygen combined with non-invasive ventilation preoxygenation for intubation in hypoxaemic patients in the intensive care unit: the single-centre, blinded, randomised controlled OPTINIV trial. Intensive Care Med 2016; 42:1877-1887

19. Ng I, Krieser R, Mezzavia P, et al: The use of Transnasal Humidified Rapid-Insufflation Ventilatory Exchange (THRIVE) for pre-oxygenation in neurosurgical patients: A randomised controlled trial. Anaesth Intensive Care 2018; 46:360-367

20. Lodenius A, Piehl J, Östlund A, et al: Transnasal humidified rapidinsufflation ventilatory exchange (THRIVE) vs. facemask breathing pre-oxygenation for rapid sequence induction in adults: a prospective randomised non-blinded clinical trial. Anaesthesia 2018; 73:564-571

21. Vourc'h M, Baud G, Feuillet F, et al: High-flow nasal cannulae versus non-invasive ventilation for preoxygenation of obese patients: The PREOPTIPOP Randomized Trial. EClinicalMedicine 2019; 13:112-119 
22. Zhao $\mathrm{H}$, Wang $\mathrm{H}$, Sun $\mathrm{F}$, et al: High-flow nasal cannula oxygen therapy is superior to conventional oxygen therapy but not to noninvasive mechanical ventilation on intubation rate: A systematic review and metaanalysis. Crit Care 2017; 21:184

23. Semler MW, Janz DR, Lentz RJ, et al; FELLOW Investigators; Pragmatic Critical Care Research Group: Randomized trial of apneic oxygenation during endotracheal intubation of the critically III. Am J Respir Crit Care Med 2016; 193:273-280

24. Maitra S, Som A, Bhattacharjee S, et al: Comparison of high-flow nasal oxygen therapy with conventional oxygen therapy and noninvasive ventilation in adult patients with acute hypoxemic respiratory failure: A meta-analysis and systematic review. J Crit Care 2016; 35:138-144

25. Ni YN, Luo J, Yu H, et al: The effect of high-flow nasal cannula in reducing the mortality and the rate of endotracheal intubation when used before mechanical ventilation compared with conventional oxygen therapy and noninvasive positive pressure ventilation. A systematic review and meta-analysis. Am J Emerg Med 2018; 36:226-233

26. Ni YN, Luo J, Yu H, et al: Can high-flow nasal cannula reduce the rate of endotracheal intubation in adult patients with acute respiratory failure compared with conventional oxygen therapy and noninvasive positive pressure ventilation?: A systematic review and meta-analysis. Chest 2017; 151:764-775

27. Cabrini L, Landoni G, Baiardo Redaelli M, et al: Tracheal intubation in critically ill patients: A comprehensive systematic review of randomized trials. Crit Care 2018; 22:6

28. Adnet F, Borron SW, Racine SX, et al: The intubation difficulty scale (IDS): Proposal and evaluation of a new score characterizing the complexity of endotracheal intubation. Anesthesiology 1997; 87:1290-1297 